the availability of antenatal diagnosis. There has not been a single case of $\beta$-thalassaemia within the Cypriot community during the past two years, as a result of intensive community education programmes and the awareness of both the community itself and those general practitioners in North London who serve it.

Parents who were at risk of having an affected child had virtually stopped reproducing before antenatal diagnosis, but now are having a normal number of children, as it can be guaranteed that all of these will be healthy. In this way, it has been shown that far from resulting in a decrease in the number of normal children being born, antenatal diagnosis with abortion of affected pregnancies has resulted in a large increase in normal children, which is what both the families at risk and the community wish.

However, antenatal diagnosis (whether using fetal blood or DNA) can only deal with the situation in advanced countries at the moment, and even in Britain, Italy and Greece there are still seriously ill children with thalassaemia. Bone marrow transplantation for blood diseases such as the leukaemias is now an established, if risky, clinical practice in several centres, and D. Thomas (University of Seattle) discussed whether this procedure might also work for thalassaemia. The best results in bone marrow transplantation are achieved with HLA-compatible brothers or sisters, and these certainly exist in thalassaemic families. However, transplantation should be carried out early, when the child is well, and not when the tissues have already been damaged by high levels of transfusion and the iron deposits this leads to. The question facing clinicians in the immediate future is whether to risk the life of a relatively well child with thalassaemia by giving a marrow transplant, knowing that if it works (a 75 per cent chance) the child will be healthy permanently - or to hope for other, less heroic treatment to be developed, knowing that if you guess wrong, the child may die in late teens of a very unpleasant disease.

\title{
Minor body mass determination
}

\section{from David W. Hughes}

SPACECRAFT missions to both asteroids and comets have been much in the news recently. One of the aims of these investigations will be the accurate determination of the masses of minor bodies in the Solar System.

From theoretical considerations, the mass of a typical short-period comet has been estimated to be about $10^{16} \mathrm{~g}$. It seems that the direct determination of a comet's mass by observing the perturbation that it induces in the orbit of another celestial body is quite out of the question. One of the closest approaches to Earth of any recorded comet was that of periodic comet Lexell, which on 1 July 1770 came to within $2.25 \times 10^{6} \mathrm{~km}$, about six times further away than the Moon. Laplace recorded in his Traité De Mécanique Céleste (Paris, 1805) that this close encounter had no measurable effect on the length of the sidereal year and thus the mass of this comet must be less than $2 \times 10^{-4}$ the mass of the Earth (which is $6 \times 10^{27} \mathrm{~g}$ ).

Asteroids are generally bigger than comets and therefore the problem is slightly easier. A great many positional observations are available over a long time period for the largest two, Ceres and Pallas, beginning with the years of discovery, 1801 and 1802 . These observations provide information about the accumulated gravitational effects which each of the two bodies have produced in the orbital motions of the other. The total effects are small. Schubart (Astr. Astrophys. 30; 289, 1974) integrated the data for 1802-1970 and found the mass of Ceres to be $(1.17 \pm 0.06) \times 10^{24} \mathrm{~g}$. Pallas was smaller - $(2.6 \pm 0.8) \times 10^{23} \mathrm{~g}$ - and so the error in its mass determination was relatively larger.

A more recent determination of the mass of a minor body has been attempted by analysing the radio-tracking data of a spacecraft as it flies close to the body. The Viking Orbiter 1 made several flybies to within $80-300 \mathrm{~km}$ of the Mars satellite Phobos. Radiometric tracking from Earth measured the spacecraft - Earth distance and the geocentric velocity of the spacecraft. The latter was achieved by recording the Doppler shift in the $S$ and $X$ band frequencies transmitted by the spacecraft. Analysis of the gravitational perturbations induced in the orbit of the spacecraft led to a mass determination of $(9.9 \pm 0.6) \times 10^{18} \mathrm{~g}$ for Phobos - an object which is about 21.5 $\mathrm{km}$ across.

The problem of measuring the mass of a comet by using the tracking data of an in situ spacecraft has been analysed by D.K. Yeomans, M. Ananda, W.L. Sjogren and L.J. Wood of the Jet Propulsion Laboratory, Pasadena (see J.astronaut. Sci. 29; 19, 1981). In a representative experiment, the authors decide to determine the mass of the short-period comet Tempel 2 during a rendezvous mission. A solar electron propulsion system (SEPS) has been used to transfer the spacecraft to the comet and the data are collected near perihelion when the comet becomes active. A single slow flyby on the sunward side of the cometary nucleus is assumed.

The main relative spacecraft-comet acceleration is due to the gravitational attraction between the two bodies. There are, however, four additional accelerations to be considered. Comets near perihelion give off a lot of gas and dust, at a rate that varies with heliocentric distance and is probably uneven, the material coming from a series of relatively short-lived active regions on the spinning cometary nucleus. The magnitude of the acceleration effect can thus vary stochastically about some mean value. It also depends on the orientation of the solar array panels, obviously

David $W$. Hughes is in the Department of Physics, University of Sheffield. being larger when these are face-on to the flux as opposed to being 'feathered'. Solar radiation pressure also influences the spacecraft and again the effect depends on the area of the spacecraft presented to the radiation flux. Radiation pressure is generally larger than the gas and dust drag but is more easily accounted for as it can be considered constant over short time periods. There is also a tidal acceleration due to the unequal distance between the spacecraft, the comet and the Sun. Finally, spurious accelerations would be present, generated by the spacecraft due to the incomplete shut down of SEPS engines, altitude and articulation control subsystem gas jets. The operation of tape recorders and resonant vibrations of large solar panels also cause problems.

Two primary measurements are taken. Doppler tracking using the Deep Space Network of tracking stations enables the velocity to be found to an accuracy of 0.1 $\mathrm{cm} \mathrm{s} \mathrm{s}^{-1}$ over a count time of $1 \mathrm{~min}$. Spacecraft-comet ranging using, for example, $\mathrm{X}$ band $(10 \mathrm{GHz})$, a pulse length of $2 \mu \mathrm{s}$ and a pulse repetition frequency of $250 \mathrm{~Hz}$ can give an accuracy of $20-30 \mathrm{~m}$ when the spacecraft is within $1,000 \mathrm{~km}$ of the cometary nucleus.

Yeomans and colleagues find that the Doppler tracking data are more useful than the spacecraft-comet ranging data when it comes to determining the mass of the comet. Accuracy is improved by having both. The sensitivity of the mass determination to the stochastic nature of the gas and dust emission indicates that an accurate on-board accelerometer would also improve the accuracy.

Returning to Tempel 2, if the rendezvous spacecraft flies by the comet at a minimum distance of $100 \mathrm{~km}$ at a velocity of $2 \mathrm{~m} \mathrm{~s}^{-1}$ and data are collected for about 8 days from both a Doppler and a ranging system, a conservative estimate is that the mass can be measured to just better than $15 \%$. 